\title{
Predicting hand movements with distributional semantics: evidence from mouse-tracking
}

\author{
Daniele Gatti ${ }^{1 *}$, Marco Marelli ${ }^{2,3}$ \& Luca Rinaldi ${ }^{1,4}$ \\ ${ }^{1}$ Department of Brain and Behavioral Sciences, University of Pavia, Pavia (Italy) \\ ${ }^{2}$ Department of Psychology, University of Milano-Bicocca, Milano, Italy \\ ${ }^{3}$ NeuroMI, Milan Center for Neuroscience, Milano, Italy \\ ${ }^{4}$ Cognitive Psychology Unit, IRCCS Mondino Foundation, Pavia (Italy)
}

* Corresponding author:

Daniele Gatti, Department of Brain and Behavioural Science, University of Pavia, Piazza Botta 6, 27100 Pavia, Italy. e-mail address: daniele.gatti@unipv.it 


\begin{abstract}
Despite mouse-tracking has been taken as a real-time window on different aspects of human decisionmaking processes, whether purely semantic information affects response conflict at the level of motor output is still unknown. Here, we investigated the possible effects of semantic knowledge on hand movements by predicting participants' performance in a mouse-tracking task through distributional semantics, a usage-based modelling approach to meaning. Participants were shown word pairs and were required to perform a two-alternative forced choice task by moving the computer mouse and selecting either the more abstract or the more concrete word, depending on the experimental condition. Results showed that mouse trajectories were affected by the semantic relatedness of the word-pairs as indexed by the distributional semantic model, despite this dimension was not relevant for the task at hand. In particular, mouse trajectories reflected the response conflict and its temporal evolution, with larger deviation and movement uncertainty for increasing word semantic relatedness. These findings testify the effects of word meaning on decision-making processes as indexed by a mouse-tracking conflict. They also document a strict interplay between sensory-motor processes and the structure of human semantic memory.
\end{abstract}

Keywords: semantic memory, distributional semantic models, mouse-tracking, semantic relatedness, hand movements. 


\section{Introduction}

In the last decade, mouse-tracking has seen enduring and rising popularity across several branches of cognitive science, mainly because of the methodological and theoretical advantages this approach carries (Stillman, Shen, \& Ferguson, 2018). As compared to reaction times, indeed, mouse-tracking has been revealed to be particularly reliable in isolating the dynamics of response conflict, by quantifying the relative directness with which participants make their decision (Freeman, 2018). That is, with mouse-tracking it is possible to quantify the stages by which the decision process unfolds (e.g., Kieslich, Henninger, Wulff, Haslbeck, \& Schulte-Mecklenbeck, 2019). Accordingly, mousetracking has been successfully used across a large number of domains such as language (Lins, \& Schöner, 2019; Spivey, Grosjean, \& Knoblich, 2005), social cognition (Freeman, Dale, \& Farmer, 2011; Freeman, Pauker, \& Sanchez, 2016) and memory (Papesh, \& Goldinger, 2012; Papesh, Hicks, \& Guevara Pinto, 2019).

Briefly, in mouse-tracking tasks, participants are generally required to move from a starting point position (i.e., typically placed in the middle-bottom part of the screen) to select either of the alternatives presented in the two upper corners of the screen. On the assumption that motor outputs (i.e., hand movements) are executed in parallel with the decision that participants are required to make (e.g., Freeman, \& Ambady, 2010), mouse-tracking packages allow to quantify the conflict of the choice and its evolution, which cannot be directly assessed using only reaction times (Stillman et al., 2018). The magnitude of the decision conflict is generally quantified by computing the maximum deviation from the direct path (i.e., the furthest point on the actual trajectory from the idealized straight trajectory between the starting point and the selected stimulus), while the decision evolution is quantified by means of sample entropy, which measures the degree of movement irregularity and unpredictability (for a complete discussion on other possible indexes see, Freeman \& Ambady, 2010; Stillman et al., 2018). More specifically, the greater the deviation from a straight path or the trajectory irregularity, the greater the decision conflict. In addition to this, mouse-tracking provides a precious real-time window into the temporal sequence of the processes subserving the conflict, being informative about the time at which the decision takes place.

Despite mouse tracking has been employed to investigate different cognitive functions, including the language domain, the decision conflict subserving word meaning processes are still largely unknown. Insofar, evidence for an effect of word meaning on response selection has been mainly observed in priming tasks, hence focusing mostly on reaction times. For instance, in lexical priming tasks, the response to a target word (e.g., dog) has been systematically shown to vary as a function of the preceding linguistic context (i.e., the prime word) (e.g., Meyer \& Schvaneveldt, 1971). Typically, the 
target word elicits a faster response after a meaning-related prime word (e.g., cat) as compared to when it is presented after an unrelated word (e.g., car). Similar evidence has been gathered from a previous mouse-tracking study, in which participants were asked to indicate which target word, among two possible alternatives, was more associated with a cue word (Hindy, Hamilton, Houghtling, Coslett, \& Thompson-Schill, 2009). Results showed that the response conflict, as indexed by the maximum deviation, was greater for more semantically associated target words as compared to less semantically associated pairs (Hindy et al., 2009). Yet, in this study semantic relatedness was operationalized on a dichotomous basis (i.e., by comparing weakly associated with strongly associated words) and participants' performance was directly predicted by this very same dimension (i.e., participants had to select the target word most semantically related to the cue).

Here, building upon this evidence, we take advantage from distributional models (DSMs) to explore the decisional stages subserving word meaning processing. DSMs induce words meanings from large databases of natural language data, representing them as high-dimensional numerical vectors. More critically, DSMs are thought to well capture the structure of semantic memory, in which word meanings would be conceived as distributed representations populating a continuous mental space (Günther, Rinaldi \& Marelli, 2019; Jones, Willits, \& Dennis, 2015). On these grounds, DSMs may be considered the ideal tool to probe whether word meanings affect not only the processes driving reaction times, but also the programming and execution of (hand) movements. Accordingly, previous studies have shown that word relatedness measures extracted from DSMs can predict lexical priming effects at the item-level (Günther, Dudschig, \& Kaup, 2016; see also Jones, Kintsch, \& Mewhort, 2006; Mandera, Keuleers, \& Brysbaert, 2017). In these studies, word meaning was indeed operationalized as a quantification of a word distribution over linguistic contexts from large corpora of natural text.

In the present study, participants were therefore shown several word pairs and were required to indicate with their mouse the more abstract one (or the more concrete one, depending on the experimental condition). We then predicted participants' responses in a two-alternative forced choice task using the degree of semantic relatedness between the two words as extracted from DSMs. Despite word relatedness was not a primary dimension for performing the task, we nevertheless expect this dimension to influence participants' performance, in line with evidence from priming experiments. In particular, we predict that the higher the semantic relatedness between the words to be judged, the higher the response conflict. This should be reflected in the specific profiles of hand movement trajectories, with a higher maximum deviation from the direct path and an higher sample entropy as a function of increasing semantic relatedness between the two words presented. 


\section{Methods}

\section{Participants}

Fifty-five students participated in the study for course credits. Fifteen participants were excluded as they used the trackpad during the experiment (see below the section Procedure). The final sample was composed by 40 participants ( 8 males, $M$ age $=22.15$ years, $S D=2.90$ ). All participants were native Italian speakers, had normal or corrected to normal vision and were naïve to the purpose of the study. Informed consent was obtained from all participants before the experiment. The protocol was approved by the psychological ethical committee of the University of Pavia and participants were treated in accordance with the Declaration of Helsinki.

\section{Distributional semantic model}

Vector representations for the words used in this study were extracted from a DSM obtained via the application of neural networks, and in particular the Continuous Bag of Words (CBOW) method, an approach originally proposed by Mikolov and colleagues (Mikolov, Chen, Corrado, \& Dean, 2013): when using CBOW, the obtained vector dimensions capture the extent to which a target word is reliably predicted by the contexts in which it appears. The model, released by Marelli (2017), was trained on itWaC, a free Italian text corpus based on web-collected data and consisting of about 1.9 billion tokens (semantic relatedness values are available online via the SNAUT database: http://meshugga.ugent.be/snaut-italian-2/2. The model used is set on the following parameters: 5word co-occurrence window, 400-dimension vectors, negative sampling with $k=10$, subsampling with $t=1 \mathrm{e}^{-5}$. This set of parameters defines the learning procedure used to induce word vectors (Mikolov, Chen et al., 2013). Co-occurrence window size indicates how large the considered lexical contexts are; in our case, a 5-word window indicates that we estimated predictions concerning 2 words on the left and 2 words on the right of the target word. From this DSM, we extracted vector representations for the words used in this study. These representations can be used to estimate the degree of semantic relatedness (SRel) between word pairs by simply considering the cosine of the angle formed by their vectors: in this way, the higher the SRel value (i.e., the closer the vectors in the multidimensional space), the more semantically related the words are expected to be as predicted by the DSM. 


\section{Stimuli}

The stimuli used in the present study were built starting from the words included in the Italian ANEW database (Montefinese, Ambrosini, Fairfield, \& Mammarella, 2014). In this study, a large number of participants had been asked to rate Italian words on different scales, including concreteness (ranging from 1 , abstract, to 9 , concrete).

First, from the ANEW database we paired all the words included and computed for each pair its difference in concreteness (i.e., by subtracting the word with the lower value of concreteness from the word with the higher value). Then, in order to allow participants in our study to easily discriminate the more concrete/abstract of the two words, we removed pairs with small difference in concreteness (i.e., concreteness difference $<2.5$ ). For each of the remaining word pairs, we extracted a SRel index based on the cosine of the angle formed by their corresponding vectors (see the previous section for a description of the DSM used). Finally, we selected 120 word-pairs guaranteeing almost uniformly distributed SRel values (see Figure 1A for SRel distribution and Figure 1B for the distribution of concreteness difference for the word pairs selected).

In particular, in the final set of 120 word pairs the concreteness difference ranged from 2.6 for the pair "poesia - penna" ("poetry - pen"), respectively with concreteness $=5.85$ and $=8.45$; to 5.4 for the pair "spirito - specchio" ("spirit - mirror"), respectively with concreteness $=3.15$ and $=8.55$; while the SRel varied from very low (SRel = .007, e.g., "idrante - intelletto"; "hydrant - intellect") to relatively high values (SRel = .39, e.g., "musica - violino"; "music - violin"). SRel and concreteness difference showed a low negative correlation, $r=-.17, p=.054$, consistent with the notion that DSM can encode concreteness-related information (Hollis \& Westbury, 2016).

A

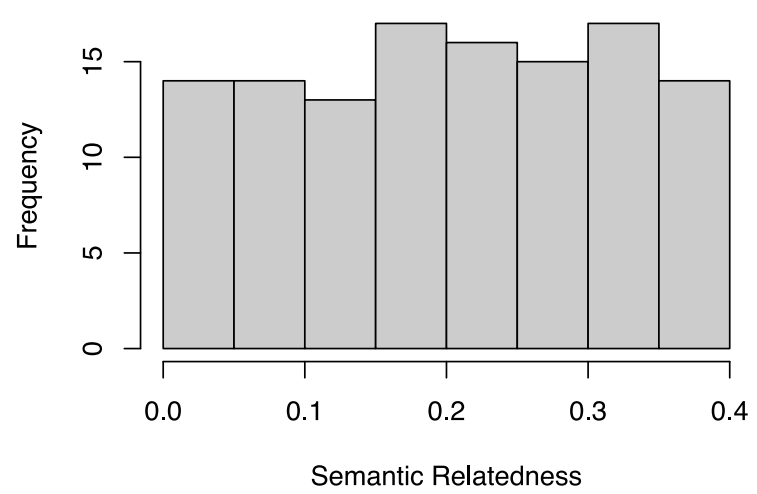

B

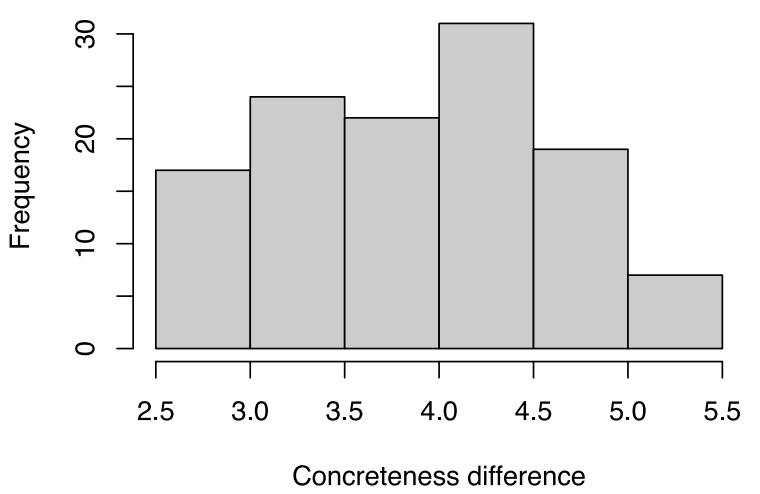

Figure 1. Frequency distributions of the Semantic Relatedness values (A) and of concreteness difference (B) of the word-pairs included in this study. Note that for 
concreteness difference the distribution starts from an high value, since in order to include only word pairs in which the correct answer was easily discriminable, we did not include word pairs with concreteness difference $<2.5$.

\section{Procedure}

Participants were tested online using Psychopy (Pierce, 2007, 2009; Pierce \& MacAskill, 2018; Pierce et al., 2019) through the online platform Pavlovia (https://pavlovia.org/).

At the beginning of the experiment, participants were asked to indicate the kind of tool with which they were answering (i.e., external mouse or trackpad). Participants were also informed that the use of the trackpad would not have affected the earning of the course credits. This allowed us to identify those participants who did not use the external mouse, who were subsequently excluded from the analyses since the reliability of mouse-tracking data collected with the trackpad is not yet established in the scientific literature. ${ }^{1}$

Then, participants were instructed on how they were supposed to use their mouse during the task, and, after a few practice trials, were asked to perform the experimental task. Participants were shown two words and were instructed to indicate the more concrete/abstract one (half of the participants was asked to indicate the more concrete word, the other half the more abstract one) using their mouse: participants had to first click on a square presented below the "START" label by pressing the mouse left button, and next to move to the selected option, again pressing the left button to make their decision. Each trial began with a fixation cross presented at the center of the screen (500 ms), then a red "START" button appeared at the bottom-center of the screen (Arial font; screen coordinates: $\mathrm{x}=$ $0, y=-.35)$; after the participants clicked on it, the two words were shown at the upper corners of the screen (Arial font; screen coordinates: $\mathrm{x}= \pm .50, \mathrm{y}=.25$ ), along with two squares that participants used as buttons (Figure 2). Participants answered to each trial by moving the mouse from the "START" button to the square associated to the chosen word and by clicking on this latter.

\footnotetext{
${ }^{1}$ Note that the effects of did not change when including also the participants who completed the task with the trackpad.
} 


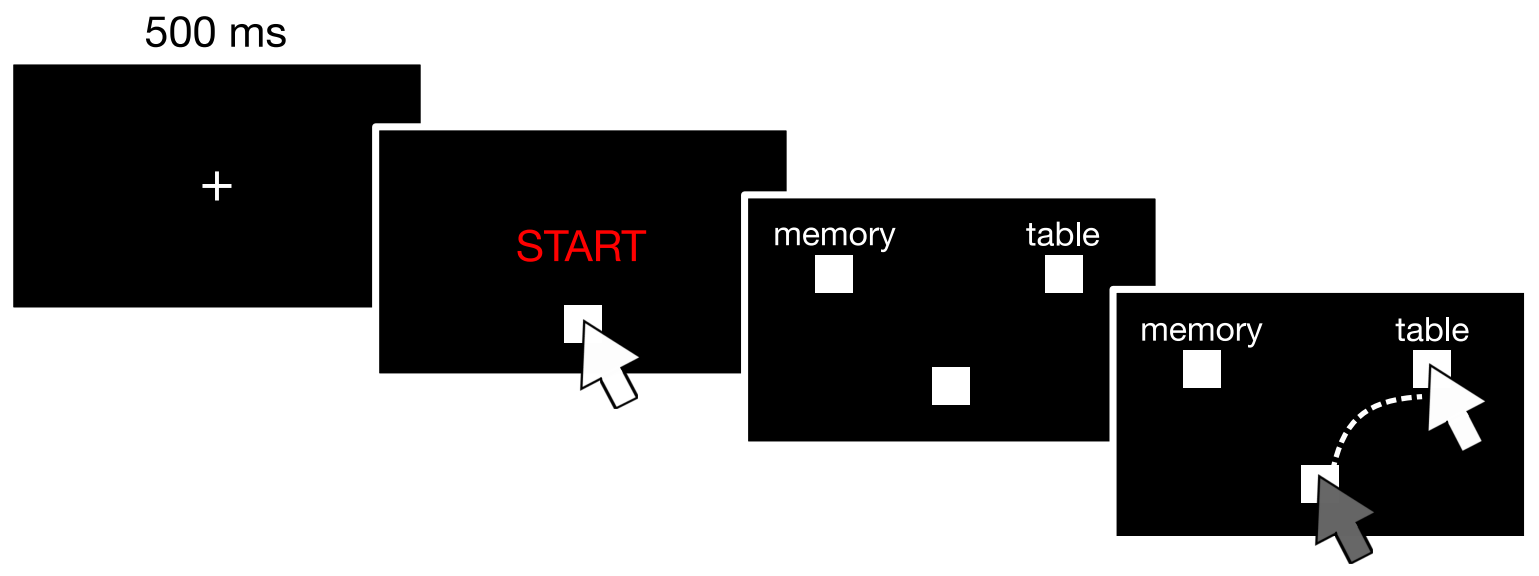

Figure 2. The timeline of the task. Participants were asked to indicate the more concrete/abstract of the two words shown using their mouse. In particular, after clicking on the square presented below the "START" signal, they were shown two words and were required to make their judgement by moving their mouse and clicking on the square below the selected word.

\section{Data analysis}

The dependent variables were maximum deviation from direct path (MD), sample entropy, initiation reaction times (RTs), maximum deviation from direct path time (MDtime) and overall RTs.

MD is defined as the furthest point on the actual trajectory from the idealized straight trajectory and is thought to quantify the conflict in the choice. Sample entropy is defined as the degree of irregularity and unpredictability in movement across the $\mathrm{x}$-axis and is thought to measure the evolution of the choice (i.e., with higher values indicating higher irregularity). Initiation RT is computed as the time elapsed between the click on the START button and the first hand-movement, while MDtime indexes the time at which the trajectory reaches the MD. These two dependent variables are considered to measure two different time windows of decision-making, with the former (initiation RTs) indicating the time at which the decisional process starts and the latter (MDtime) indicating the time at which a decision is finally achieved (see for a complete discussion regarding mouse-tracking variables: Stillman et al., 2018). Finally, overall RT is computed as the time elapsed between the click on the START button and the click on the selected stimulus. This variable is therefore necessarily influenced by MDtime, in that trails in which the decision occurs later (i.e., higher MDtime) will also result in higher overall RTs. 
All the dependent variables were computed only on the trials in which participants answered correctly, using the mousetrap package (Kieslich, et al., 2019). All trajectories were normalized into 101 time-steps and remapped symmetrically in order to allow for direct comparison of trajectories which differed in duration and number of data points. Trials in which MD was \pm 3 SD from the mean of the participants were removed from the analysis (.4\% of the trials excluded). Initiation RTs, MDtime and overall RTs were all log-transformed.

The statistical analyses were performed using R-Studio (RStudio Team, 2015). We estimated five separated linear mixed models (LMMs), one for each dependent variable, with the lme4 $\mathrm{R}$ package (Bates, Maechler, Bolker, \& Walker, 2015). The graphs reported were obtained using the effects R package (Fox, 2003; Fox \& Weisberg, 2019). For each LMM, we included SRel, concreteness difference (i.e., the difference between the concreteness of the two words presented, as extracted from the Italian ANEW database; Montefinese, Ambrosini, Fairfield, \& Mammarella, 2014) and frequency difference (i.e., the absolute difference between the raw frequencies of the two words presented, as extracted from the Italian SUBTLEX; http://crr.ugent.be/subtlex-it/) as continuous predictors, while subjects and items were included as random intercepts. In particular, in lme 4 syntax, the model tested was:

$$
D V \sim \text { SSim }+ \text { delta_concreteness }+ \text { delta_frequency }+(1 \mid \text { Participant })+(1 \mid \text { Item })
$$

where the DV could be either MD, sample entropy, initiation RTs, MDtime or overall RTs. Then, for each model tested, we performed a backward selection. This procedure selects the best fitting model dropping one fixed effect at a time. To exclude the impact of overly influential outliers, after having fitted the model, data points were removed on the basis of a threshold of $2.5 S D$ standardized residual errors (model criticism; Baayen, 2008). Results based on the refitted models are reported.

\section{Results}

Non-accurate trials (151 trials on a total of 4800 trials) and trials in which overall RTs were faster than $300 \mathrm{~ms}$ or slower than $3000 \mathrm{~ms}$ (306 additional trials on a total of 4800 trials) were excluded from the analysis. Aberrant movements were detected in 236 of the 4343 remaining trials and were discarded. Overall participants' accuracy was very high $(M=.97, S D=.03)$. Descriptive statistics for the five variables are reported in Table 1. 
The correlation matrix between the five dependent variables is reported in Table 2. Overall, the correlations ranged from very low, as the one between MD and MDtime ( $r=-.04)$, to high, as the one between overall RTs and MDtime $(r=.67)$. As discussed previously, this latter correlation corroborates the fact that overall RTs is necessarily influenced by MDtime, since trials in which the decision occurs later (i.e., higher MDtime) will also result in higher overall RTs. Note that the correlations were computed on raw data, hence on a total of 4107 trials.

Table 1. Descriptive statistics for the five dependent variables analyzed. The descriptive statistics of the three variables expressing temporal processes (initation RTs, MDtime, overall RTs) are reported in milliseconds.

\begin{tabular}{r|ccccc}
\hline & MD & Sample entropy & Initiation RTs & MDtime & Overall RTs \\
\hline Mean & .32 & .12 & 320 & 870 & 1660 \\
SD & .17 & .04 & 220 & 220 & 260 \\
Min - Max & $-.02-.70$ & $.05-.20$ & $80-1180$ & $520-1550$ & $1150-2170$ \\
\hline
\end{tabular}

Table 2. Correlation matrix between the five dependent variables included in the current study. The correlation values ranged from very low to high (4105 degrees of freedom, all $p \mathrm{~s}<.01)$.

\begin{tabular}{c|ccccc}
\hline & MD & Sample entropy & Initiation RTs & MDtime & Overall RTs \\
\hline MD & 1 & & & & \\
Sample entropy & .61 & 1 & & & \\
Initiation RTs & -.31 & -.29 & 1 & 1 & \\
MDtime & -.04 & .05 & .46 & .67 & 1 \\
Overall RTs & .27 & .34 & .20 & \\
\hline
\end{tabular}

For all the five models tested except for the one on initiation RTs, the backward selection dropped both concreteness difference and frequency difference from the model. Hence, the final model included only SRel as predictor. The backward selection on the model on initiation RTs dropped all the predictors indicating that the null model was the best one.

The LMM on MD showed a significant effect of SRel, $F(1,114.9)=8.01, p=.005$, Pseudo- $R^{2}$ (total) $=.29, \beta=.19$. In particular, the higher the SRel, the higher the MD (see Figure 3 for a graphical representation of the actual trajectories collapsed as a function of three different levels of SRel). 
The LMM on sample entropy showed as well a significant effect of SRel, $F(1,113.22)=3.93, p=$ .03, Pseudo- $R^{2}($ total $)=.26, \beta=.02$. In particular, the higher the SRel, the higher the sample entropy. The LMM on MDtime (transformed in their logarithmic values) showed a significant effect of SRel, $F(1,116.55)=7.43, p=.007$, Pseudo- $R^{2}($ total $)=.45, \beta=.17$. In particular, the higher the SRel, the higher the MDtime.

Finally, the LMM on overall RTs (transformed in their logarithmic values) showed a significant effect of SRel, $F(1,113.73)=10.28, p=.001, P$ seudo- $R^{2}($ total $)=.46, \beta=.20$. In particular, the higher the SRel, the higher the RTs.

A

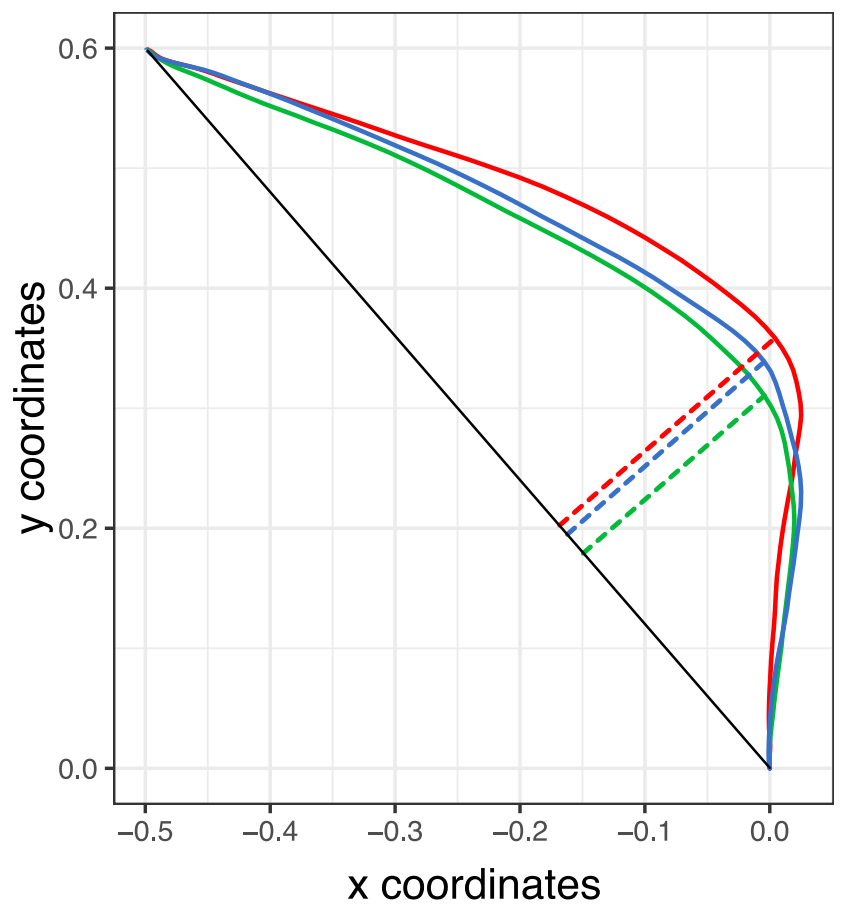

B

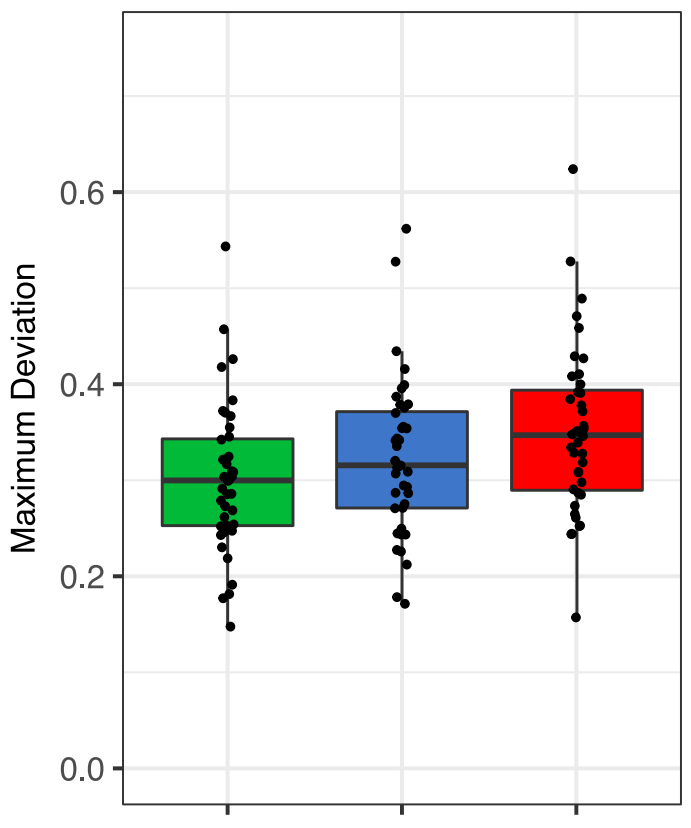

Semantic Relatedness

苗 Low

Figure 3. Schematic representation of how the meaning distance (in dashed lines), computed from the direct path (black straight line) to the maximum deviation registered, varied across three levels of SRel. Note that in order to represent as categorical the continuous predictor, we divided it in three arbitrary categories, each comprising 40 wordpairs. (A). A boxplot of the maximum deviation for each word-pair across the three hypothetical levels of SRel (B). 


\section{Discussion}

In the present study, we investigated the impact of word meaning on decision-making processes during a two-alternative forced choice concrete-abstract judgement task with a mouse-tracking approach. In particular, we combined data from distributional semantic models and from a mousetracking task to shed light on the response conflict driving word meaning processing. Participants were shown several word-pairs and were required to indicate the more abstract word (or more concrete one) using the computer mouse. The semantic relatedness between the two words was manipulated in order to test its possible influence on decision-making processes. Our findings indicated that participants' judgements were affected by the semantic relatedness of the two words, despite this not being a dimension directly involved in performing the task at hand. Such a pattern was not captured by simple initiation reaction times, but rather in terms of the conflict driving the choice as well as its (temporal) evolution, as evidenced by the measures indexing the online processes subserving task performance. Although DSM are known to encode concreteness, up to a certain level (Hollis \& Westbury, 2016), this piece of information does not overlap with semantic relatedness: related words, in fact, does not necessarily manifest the same degree of concreteness (church vs. religion, flag vs. patriotism). Accordingly, in our analyses (1) we observe a very low correlation between concreteness difference and SRel, and (2) concreteness difference is dropped from model selection, for all the dependent variables tested. The results, therefore, seem to genuinely depend on the relatedness between word meaning, in a way that is largely independent form their concreteness level (notwithstanding participants were asked to perform a concreteness-judgment task): the more the competitor word will be related to the target word, the more the response will be affected in terms of decision conflict. This indicates that semantic relatedness can affect human behavior well beyond processing speed, testifying its influence also on online response conflict at the level of motor control. While the impact of semantic relatedness as extracted from DSMs on reaction times has been already established (Günther et al., 2016; see also Jones et al., 2006; Mandera et al., 2017), our study is the first to report its effect also on mouse-tracking variables. Indeed, previous studies have shown that semantic relatedness facilitates participants' reaction times, with faster responses occurring for more semantically related word-pairs (Günther, et al., 2016). Here, we found a similar pattern for the overall reaction times, an index that takes into account the overall time window (i.e., from the movement initiation to the selection of the target word), hence also being possibly affected by the conflict arising during the evaluation process. Indeed, the other variables considered (namely MD, sample entropy and MDtime) were particularly informative of decision-making processes. That is, the results of the maximum deviation index suggest that the conflict increased with increasing semantic relatedness, as the furthest point on the actual trajectory was farther from the idealized 
straight trajectory for larger SRel values (Freeman, 2018). Analogously, the results of sample entropy indicate that participants' hand trajectories were more spatially complex (i.e., showed higher unpredictability; Freeman, 2018), with increasing semantic relatedness. The moderate correlation between MD and sample entropy may nevertheless suggest that the effects observed on the latter variable may be partially determined by the former, in that the maximum deviation from the direct path would naturally affect also movement irregularity. Notably, regarding the time course of participants' decisions, our findings also show that the effects of semantic relatedness was relevant only in the online component of the choice (i.e., on MDtime) and in the overall RTs. Indeed, MDtime results suggest that the time at which a decision occurred was delayed for more semantically similar words. Such an effect may have likely determined also the difference observed in terms of overall RTs, a variable that is necessarily influenced by MDtime (i.e., trails in which the decision occurs later will also result in higher overall RTs), as also observed in the moderate correlation between them. On the contrary, no effect was detectable on initiation reaction times: this indicates that, at this initial stage, the participants did not yet elaborate the two stimuli in terms of semantic relatedness. This last finding also corroborates the need of a mouse tracking approach for better grasping deep semantic memory processes.

We also pinpoint that the different direction of the effects across our study (i.e., higher semantic relatedness interfering with participants' performance) and lexical priming effects reported by previous studies (i.e., higher semantic relatedness facilitating participants' performance) can be easily explained by the different requirements imposed by the tasks adopted. That is, while in lexical priming tasks a semantically similar prime pre-activated the meaning of the target word, here the higher shared meaning across the two to-be-compared words made in turn more difficult the primary judgment that participants had to make (i.e., indicating the abstract/concrete word).

Our findings have therefore relevant implications from both theoretical and methodological points of view. On a theoretical level, our data clarify the participation of semantic memory when the performance is measured through fine-grained hand movements. This adds to previous evidence showing that distributional semantic models are highly performing across a wide range of semantic tasks, such as multiple-choice tests (Bullinaria \& Levy, 2012), word categorization (Baroni \& Lenci, 2010), word relatedness ratings (Bruni, Tran, \& Baroni, 2014), word naming and lexical decision (Marelli \& Amenta, 2018), as well as recognition memory (Gatti, Rinaldi, Marelli, Mazzoni, \& Vecchi, in press), providing in turn further support to the idea that distributional semantic models are indeed extremely efficient in capturing the structure of human semantic memory (Günther et al., 2019). On a methodological level, the fact that distributional semantic models paired with mouse- 
tracking can be used to investigate deep decision-making features of human behavior, open new avenues for probing the detailed processes subserving the mechanisms of semantic memory.

Finally, the present findings can also be interpreted within the theoretical debate regarding the interplay between sensory-motor and cognitive processing. While standard disembodied theories proposed that cognitive processes are essentially amodal and are structurally detached from perceptual, motor, and emotional systems (e.g., Fodor, 1975; Pylyshyn, 1984), embodied perspectives have rather proposed that different aspects of human cognition -including word meaning- are grounded on sensory-motor interactions (e.g., Barsalou, 1999; Glenberg, 1997). In the halfway between these proposals, others have maintained that conceptual and sensory-motor representations are distinct but interact on different levels (e.g., Binder \& Desai, 2011; Damasio, 1989; Patterson, Nestor, \& Rogers, 2007). The present findings are consistent with this interdependent perspective, by showing that the structure of human semantic memory can affect complex hand movements such as those measured with mouse tracking.

In conclusion, using distributional semantic models paired with mouse-tracking, we outlined a datadriven framework to account for semantic memory involvement in decision-making as measured by hand movements. Our findings well complement previous evidence accounting for participants' behavior in semantic tasks and provide insights on the impact of semantic memory on cognitive operations. 


\section{Declarations}

\section{Conflicts of interest}

The authors declare no conflicts of interest.

\section{Funding}

This work was supported by funding from the Italian Ministry of Health (Ricerca Corrente 2021) to LR.

\section{Data availability}

The data used in this study are reported as Supplementary Materials. 


\section{References}

Baayen, R.H. (2008). Analyzing linguistic data: A practical introduction to statistics using $R$. Cambridge: Cambridge University Press.

Baroni, M., \& Lenci, A. (2010). Distributional memory: A general framework for corpus-based semantics. Computational Linguistics, 36, 673-721.

Barsalou, L.W. (1999). Perceptual symbol systems. Behavioral and Brain Sciences, 22(4), 577-609.

Bates, D., Maechler, M., Bolker, B., \& Walker, S. (2015). Fitting Linear Mixed-Effects Models Using Ime4. Journal of Statistical Software, 67(1), 1-48.

Binder, J.R., \& Desai, R.H. (2011). The neurobiology of semantic memory. Trends in Cognitive Sciences, 15(11), 527-536.

Bruni, E., Tran, N.K., \& Baroni, M. (2014). Multimodal distributional semantics. Journal of Artificial Intelligence Research, 49, 1-47.

Bullinaria, J.A., \& Levy, J.P. (2007). Extracting semantic representations from word co-occurrence statistics: A computational study. Behavior Research Methods, 39, 510-526.

Damasio, A. R. (1989). Time-locked multiregional retroactivation: A systems-level proposal for the neural substrates of recall and recognition. Cognition, 33(1-2), 25-62.

Fodor, J. A. (1975). The language of thought. Harvard University Press.

Fox, J. (2003). Effect Displays in R for Generalised Linear Models. Journal of Statistical Software, 8(15), 1-27. URL: http://www.jstatsoft.org/v08/i15/

Fox, J., \& Weisberg, S. (2019). An R Companion to Applied Regression, 3rd Edition. Thousand Oaks, $\mathrm{CA}<\mathrm{http}: / /$ tinyurl.com/carbook $>$

Freeman, J.B. (2018). Doing psychological science by hand. Current Directions in Psychological Science, 27(5), 315-323.

Freeman, J.B., \& Ambady, N. (2010). MouseTracker: Software for studying real-time mental processing using a computer mouse-tracking method. Behavior Research Methods, 42(1), 226-241.

Freeman, J.B., Dale, R., \& Farmer, T. (2011). Hand in motion reveals mind in motion. Frontiers in Psychology, 2, 59.

Freeman, J.B., Pauker, K., \& Sanchez, D.T. (2016). A perceptual pathway to bias: Interracial exposure reduces abrupt shifts in real-time race perception that predict mixed-race bias. Psychological Science, 27(4), 502-517.

Gatti, D., Rinaldi, L., Marelli, M., Mazzoni, G., \& Vecchi, T. (in press). Decomposing the semantic processes underpinning veridical and false memories. Journal of Experimental Psychology: General.

Glenberg, A.M. (1997). What memory is for. Behavioral and Brain Sciences, 20(1), 1-55. 
Günther, F., Dudschig, C., \& Kaup, B. (2016). Latent semantic analysis cosines as a cognitive similarity measure: Evidence from priming studies. The Quarterly Journal of Experimental Psychology, 69, 626-653.

Günther, F., Rinaldi, L., \& Marelli, M. (2019). Vector-space models of semantic representation from a cognitive perspective: A discussion of common misconceptions. Perspectives on Psychological Science, 14(6), 1006-1033.

Hindy, N.C., Hamilton, R., Houghtling, A.S., Coslett, H.B., \& Thompson-Schill, S.L. (2009). Computer-mouse tracking reveals TMS disruptions of prefrontal function during semantic retrieval. Journal of Neurophysiology, 102(6), 3405-3413.

Hollis, G., \& Westbury, C. (2016). The principals of meaning: Extracting semantic dimensions from co-occurrence models of semantics. Psychonomic Bulletin \& Review, 23(6), 1744-1756.

Jones, M.N., Kintsch, W., \& Mewhort, D.J.K. (2006). High-dimensional semantic space accounts of priming. Journal of Memory and Language, 55, 534-552.

Kieslich, P.J., Henninger, F., Wulff, D.U., Haslbeck, J.M.B., \& Schulte-Mecklenbeck, M. (2019). Mouse-tracking: A practical guide to implementation and analysis. In M. SchulteMecklenbeck, A. Kühberger, \& J. G. Johnson (Eds.), A Handbook of Process Tracing Methods (pp. 111-130). New York, NY: Routledge.

Lin, Y.C., \& Lin, P.Y. (2016). Mouse tracking traces the "Camrbidge Unievrsity" effects in monolingual and bilingual minds. Acta Psychologica, 167, 52-62.

Lin, Y.C., \& Lin, P.Y. (2020). Reading minds in motion: Mouse tracking reveals transposed-character effects in Chinese compound word recognition. Applied Psycholinguistics, 41(4), 727-751.

Lins, J., \& Schöner, G. (2019). Computer mouse tracking reveals motor signatures in a cognitive task of spatial language grounding. Attention, Perception, \& Psychophysics, 81(7), 2424-2460.

Mandera, P., Keuleers, E., \& Brysbaert, M. (2017). Explaining human performance in psycholinguistic tasks with models of semantic similarity based on prediction and counting: A review and empirical validation. Journal of Memory and Language, 92, 57-78.

Marelli, M. (2017). Word-embeddings Italian Semantic Spaces: A semantic model for psycholinguistic research. Psihologija, 50(4), 503-520.

Marelli, M., \& Amenta, S. (2018). A database of orthography-semantics consistency (OSC) estimates for 15,017 English words. Behavior Research Methods, 50(4), 1482-1495.

Meyer, D.E., \& Schvaneveldt, R.W. (1971). Facilitation in recognizing pairs of words: evidence of a dependence between retrieval operations. Journal of Experimental Psychology, 90(2), 227

Mikolov, T., Chen, K., Corrado, G., \& Dean, J. (2013). Efficient estimation of word representations in vector space. Retrieved from https://arxiv.org/abs/1301.3781.

Montefinese, M., Ambrosini, E., Fairfield, B., \& Mammarella, N. (2014). The adaptation of the affective norms for English words (ANEW) for Italian. Behavior Research Methods, 46(3), 887-903.

Papesh, M.H., \& Goldinger, S.D. (2012). Memory in motion: Movement dynamics reveal memory strength. Psychonomic Bulletin \& Review, 19(5), 906-913. 
Papesh, M.H., Hicks, J.L., \& Guevara Pinto, J.D. (2019). Retrieval dynamics of recognition and rejection. Quarterly Journal of Experimental Psychology, 72(9), 2328-2341.

Patterson, K., Nestor, P.J., \& Rogers, T.T. (2007). Where do you know what you know? The representation of semantic knowledge in the human brain. Nature Reviews Neuroscience, 8(12), 976-987.

Peirce, J.W. (2007). PsychoPy - Psychophysics software in Python. Journal of Neuroscience Methods, 162 (1-2), 8-13.

Peirce, J.W. (2009). Generating stimuli for neuroscience using PsychoPy. Frontiers in Neuroinformatics, 2 (10), 1-8.

Peirce, J.W., \& MacAskill, M.R. (2018). Building Experiments in PsychoPy. London: Sage.

Peirce, J.W., Gray, J.R., Simpson, S., MacAskill, M.R., Höchenberger, R., Sogo, H., Kastman, E., Lindeløv, J. (2019). PsychoPy2: experiments in behavior made easy. Behavior Research Methods, 51(1), 195-203.

Pylyshyn, Z. W. (1984). Computation and cognition. MIT Press.

RStudio Team (2015). RStudio: Integrated Development for R. RStudio, Inc., Boston, MA URL http://www.rstudio.com/.

Spivey, M. J., Grosjean, M., \& Knoblich, G. (2005). Continuous attraction toward phonological competitors. Proceedings of the National Academy of Sciences, 102(29), 10393-10398.

Stillman, P.E., Shen, X., \& Ferguson, M.J. (2018). How mouse-tracking can advance social cognitive theory. Trends in Cognitive Sciences, 22(6), 531-543.

Yamamoto, N., Incera, S., \& McLennan, C.T. (2016). A reverse stroop task with mouse tracking. Frontiers in Psychology, 7, 670. 